\title{
Synthesis of enantiopure 1,3-oxazolidin-2-ones from $\alpha$-dibenzylamino esters
}

\author{
Adrián Ochoa-Terán and Ignacio. A. Rivero* \\ Centro de Graduados e Investigación en Química del Instituto Tecnológico de Tijuana, \\ Apartado Postal 1166, Tijuana, B. C. 22000 México \\ E-mail: irivero@tectijuana.mx
}

\begin{abstract}
A new method to obtain enantiopure 5-substituted 1,3-oxazolidin-2-ones $\mathbf{1}$ from $\alpha$ dibenzylamino esters $\mathbf{2}$ is reported. This methodology is based on the Lewis acid catalyzed stereoselective addition of trimethylsilyl cyanide to chiral $\alpha$-dibenzylamino aldehydes 3 . Magnesium chloride and zinc iodide were tested to catalyze the addition, obtaining higher stereoselectivity with zinc iodide than with magnesium chloride.
\end{abstract}

Keywords: Enantiopure, 1,3-oxazolidin-2-ones, stereoselective addition

\section{Introduction}

In 1981, 4-substituted 1,3-oxazolidin-2-ones were introduced into organic synthesis as chiral auxiliaries by Evans. ${ }^{1}$ Since then they have been used in many reactions. These compounds are mainly prepared from the cyclization of chiral amino alcohols derived from nonracemic amino acids. Usually, $N$-acyloxazolidinones participate in stereoselective processes as alkylations, $\alpha$ substitution reactions, aldol reactions, conjugate additions and pericyclic reactions. The majority of these reactions are performed in the presence of a metal ion. ${ }^{2}$

Meanwhile, a group of 5-substituted 1,3-oxazolidin-2-ones, typified by linezolid (4) and eperozolid (5), represent a new class of synthetic antibacterial agents with potent activity against clinically important susceptible and resistant Gram-positive and anaerobic pathogens. ${ }^{3}$ This class of compounds has a novel mechanism of action that shows selective and unique binding to 50S ribosomal subunit, inhibiting bacterial translation at the initiation phase of protein synthesis. ${ }^{4}$ Due to its protein synthesis inhibitory activity these compounds are used against methicilline or vancomicin-resistant staphylococci, streptococci and enterococci bacteria that causes skin and soft tissue infections and pneumonia. ${ }^{5}$ Moreover, there are many reports for the synthesis of new

\footnotetext{
${ }^{*}$ Corresponding author. Tel.: 52-664-623-3772; Fax: 52-664-623-4043;
} 
compounds structurally related to linezolid that avoid bacterial resistance against these new antibiotics. ${ }^{6}$

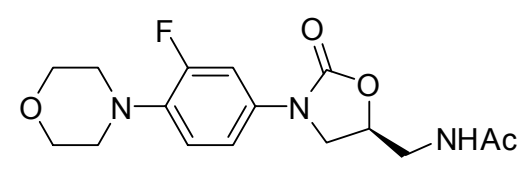

4

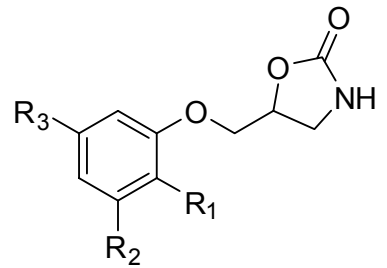

6: $\mathrm{R}_{1}=\mathrm{OMe} \mathrm{R}_{2}=\mathrm{R}_{3}=\mathrm{H}$

7: $\mathrm{R}_{1}=\mathrm{H} \quad \mathrm{R}_{2}=\mathrm{R}_{3}=\mathrm{Me}$

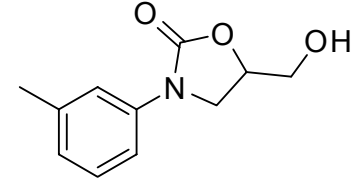

8

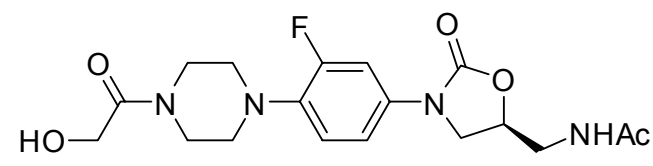

5

Mephenoxalone (6) and metaxalone (7) are 5-aryloxymethyl-1,3-oxazolidin-2-one type compounds, which have shown activity as interneuron blocking agents or depressants of central synaptic transmission. They are generally antagonists of strychnine convulsions and have been used as skeletal muscle relaxants, anticonvulsants, and tranquilizers. ${ }^{7}$ These products are prescribed to rest, physical therapy, and for the relief of discomfort associated with acute, painful musculoskeletal conditions. ${ }^{8}$ Also, there are 5-substituted 1,3-oxazolidin-2-ones, which are a new class of reversible and selective third-generation of potent and selective monoamine oxidase type A (MAO) inhibitors as toloxatone (8; Humoryl $\left.{ }^{\circledR}\right)$, cimoxatone $(\mathbf{9})$ and befloxatone (10) which are indicated in the treatment of several neurological diseases. ${ }^{9}$ Therefore, the synthesis of chiral 1,3-oxazoldin-2-ones is an important field of research due to the potential as chiral inductors or biological active substances. ${ }^{10}$

Reetz and coworkers reported the stereoselective cyanosilylation of non-racemic $\alpha$ dibenzylamino aldehydes 3 by the addition of trimethylsilyl cyanide with different inorganic salts as Lewis acid catalysts. ${ }^{11}$ The application of some organometallic compounds have also been reported. ${ }^{12}$ These silylated intermediates are important building blocks in the synthesis of pharmacologically active compounds, ${ }^{13}$ but to the best of our knowledge, the synthesis of 1,3oxazolidin-2-ones from these silylated intermediates has yet to be reported. Only the $N$-benzoyl1,3-oxazolidin-2-one derivative from L-phenylalanine has been synthesized from a ring-closure reaction of $\beta$-hydroxyalkyl phenyl selenides through intramolecular displacement of the phenylselenoyl group with nitrogen nucleophiles. ${ }^{14}$ Previously, we have explored the construction of 1,3-oxazolidin-2-one ring from a silylated intermediate, ${ }^{15}$ as well as the stereoselective synthesis of new compounds with potential biological activity by transformation of chiral $\alpha$-amino esters. ${ }^{16}$ In this work, we have developed a new methodology for the synthesis 
of enantiopure 5-substituted 1,3-oxazolidin-2-ones from chiral silylated intermediates prepared from enantiopure $\alpha$-dibenzylamino esters.

\section{Results and Discussion}

$N, N$-Dibenzylamino esters 2 were prepared from L-phenylalanine, L-alanine, L-valine, L-leucine and L-isoleucine under standard procedures to generate $\mathbf{2 a - e , ~ r e s p e c t i v e l y , ~}{ }^{17}$ and then reduced with lithium borohydride at reflux temperature in THF, to obtain amino alcohols 11 a-e in $44 \%$ to $99 \%$ yield (Scheme 1). $\alpha$-Dibenzylamino aldehydes 3a-e were obtained in very good yields ( $83 \%$ to $98 \%$ ) by oxidation of compounds 11a-e, respectively, in Swern oxidation conditions. The stereoselective anti addition of trimethylsilyl cyanide to the $\alpha$-dibenzylamino aldehydes $\mathbf{3}$ was achieved using zinc iodide $\left(\mathrm{ZnI}_{2}\right)$ or magnesium chloride $\left(\mathrm{MgCl}_{2}\right)$ in dichloromethane at $0{ }^{\circ} \mathrm{C}$ to give the corresponding trimethylsilylcyanohydrins 12a-e in $72 \%$ to $99 \%$ yield. When $\mathrm{MgCl}_{2}$ was used, two molar equivalents of TMSCN were required to complete the reaction.

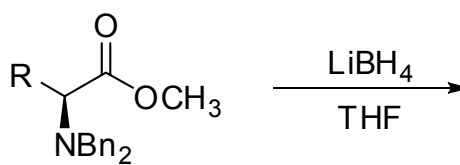

2
a: $\mathrm{R}=\mathrm{Phe}$
b: $R=M e$
c: $\mathrm{R}=i \mathrm{Pr}$
$\mathrm{d}: \mathrm{R}=i s o \mathrm{Bu}$
e: $R=\sec B u$

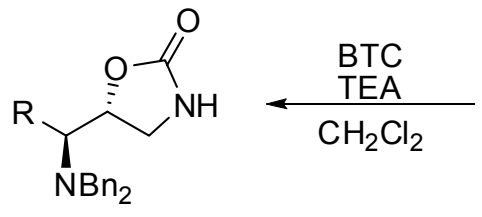

1
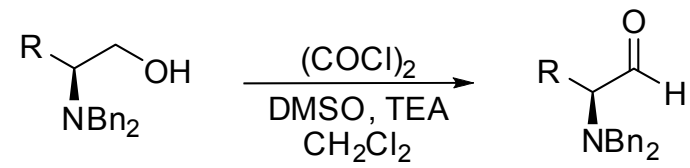

11
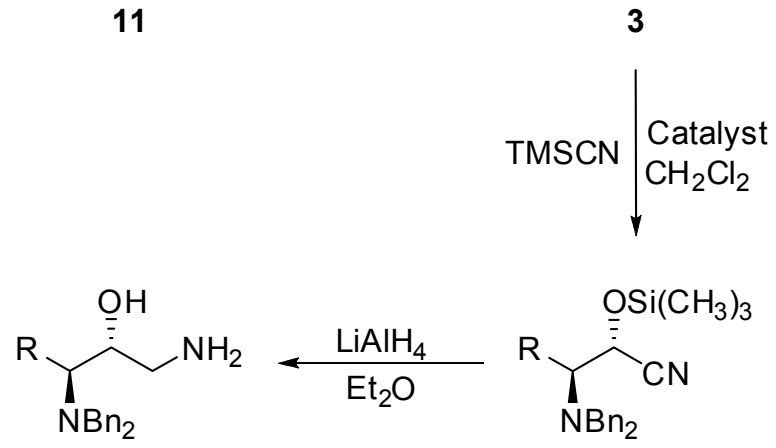<smiles>[R]C([NH3+])[C@@H](C#N)O[SiH3]</smiles>

13

12

Scheme 1. Synthesis of 5-substituted 1,3-oxazolidin-2-ones 1.

Silylated cyanohydrins 12a-e were reduced with lithium aluminum hydride in diethyl ether at $0{ }^{\circ} \mathrm{C}$ to obtain the corresponding $\alpha$-dibenzylamino alcohols 13a-e in $77 \%$ to $94 \%$ yield. Triphosgene (BTC) was used for the cyclization of amino alcohols 13a-e in dichloromethane at room temperature with yields of $35 \%$ to $73 \%$. 1,3-Oxazolidin-2-ones 1a-e were obtained in moderate overall yields ( $15 \%$ to $64 \%$, Table 1$)$. Two reaction steps were critical in this synthesis; for the reduction of amino esters 2 a-e different reaction times and yields were observed for each amino ester. Those with bulkier $\mathrm{R}$ substituent required longer reaction times and were obtained 
in lower yield in the order secbutyl<isopropyl< isobutyl. A similar effect was observed in the cyclization of amino alcohols 13a-e with BTC to form 1,3-oxazolidin-2-ones 1a-e.

Table 1. Yields in the synthesis of 5-substituted 1,3-oxazolidin-2-ones

\begin{tabular}{cccccc}
\hline Compound & Bn & Me & isoPr & isoBu & secBu \\
\hline $\mathbf{1 1}$ & 99 & 94 & 60 & 76 & 44 \\
$\mathbf{3}$ & 95 & 83 & 97 & 98 & 96 \\
$\mathbf{1 2}$ & 99 & 97 & 82 & 94 & 72 \\
$\mathbf{1 3}$ & 94 & 81 & 92 & 89 & 77 \\
$\mathbf{1}$ & 73 & 67 & 35 & 62 & 66 \\
Overall yield & 64 & 41 & 15 & 39 & 16 \\
\hline
\end{tabular}

The stereoselectivity in the synthesis of silylated cyanohydrins 12a-e was established by proton NMR chemical shifts and coupling constants. For the trimethylsilylcyanohydrin 12a synthesized using $\mathrm{ZnI}_{2}$ as catalyst, a doublet signal at $4.46 \mathrm{ppm}$ with $J=6.0 \mathrm{~Hz}$ was assigned to the hydrogen on the new chiral center. When the trimethylsilyl group was removed under mild acid conditions to afford the corresponding cyanohydrins compound, ${ }^{11 \mathrm{~d}}$ the proton NMR signal for the same hydrogen was observed at $4.00 \mathrm{ppm}$ with a $J=5.4 \mathrm{~Hz}$. Comparing these values with those reported in literature, this compound was found to be the anti stereoisomer. ${ }^{12 a}$ When trimethylsilylcyanohydrin 12a was prepared using $\mathrm{MgCl}_{2}$ as catalyst, in the proton NMR spectra was observed, in a minor proportion, a doublet signal at $4.34 \mathrm{ppm}$ with $J=4.0 \mathrm{~Hz}$, which was assigned to the hydrogen on the new chiral center for the syn stereoisomer. The addition was completely stereoselective with $\mathrm{ZnI}_{2}$, but with $\mathrm{MgCl}_{2}$ a mixture of stereoisomers was obtained (Table 2, entries 1 and 2). For compounds $\mathbf{1 2 b}$-e only the anti stereoisomer was obtained when the addition was catalyzed with $\mathrm{ZnI}_{2}$.

The stereoselectivity observed in the addition of trimethylsilyl cyanide to $\alpha$-dibenzylamino aldehydes 3 was explained in terms of a non-chelating control mechanism proposed by Reetz, ${ }^{1 \mathrm{a}, 11 \mathrm{~d}}$ where the metal ion coordinates with the carbonyl group, but not with the the $\alpha$ dibenzylamino. Also, the addtion resulted to be in line with the Felkin-Ahn model (Figure 1), where the nucleophile approaches in an orthogonal position to the less hindered face of the carbonyl group and opposite to the dibenzylamino group resulting in an anti addition selectivity. 
Table 2. Addition of trimethylsilyl cyanide to $\alpha$-dibenzylamino aldehydes 3<smiles>[R]C(CCCCCCC)C(=O)c1ccccc1</smiles>

3<smiles>[R]C(N)C(C#N)O[Si]</smiles>

syn-12<smiles>[R]C([NH3+])[C@H](C)O[SiH3]</smiles>

anti-12

\begin{tabular}{cccccc}
\hline Entry & Compound & $\mathrm{R}$ & Catalyst & Yield (\%) & syn:anti \\
\hline 1 & 12a & $\mathrm{Bn}$ & $\mathrm{ZnI}_{2}$ & 99 & $1:>99$ \\
2 & $\mathbf{1 2 a}$ & $\mathrm{Bn}$ & $\mathrm{MgCl}_{2}$ & 98 & $34: 66$ \\
3 & $\mathbf{1 2 b}$ & $\mathrm{Me}$ & $\mathrm{ZnI}_{2}$ & 97 & $1:>99$ \\
4 & $\mathbf{1 2 c}$ & $i \mathrm{Pr}$ & $\mathrm{ZnI}_{2}$ & 82 & $1:>99$ \\
5 & $\mathbf{1 2 d}$ & $i \mathrm{Bu}$ & $\mathrm{ZnI}_{2}$ & 94 & $1:>99$ \\
6 & $\mathbf{1 2 e}$ & $s \mathrm{Bu}$ & $\mathrm{ZnI}_{2}$ & 72 & $1:>99$ \\
\hline
\end{tabular}

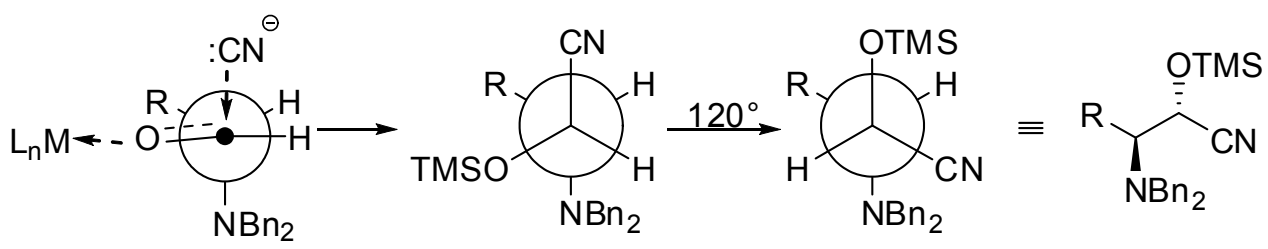

Figure 1. Felkin-Ahn model for the addition of TMSCN to aldehydes 3.

Finally, the configuration of 1,3-oxazolidin-2-ones 1a-e was confirmed by the correlations of ${ }^{1} \mathrm{H}$ NMR coupling constants, COSY and NOESY experiments. In COSY experiments, the H-5 hydrogen ( $4.83 \mathrm{ppm}, J=8.6 \mathrm{~Hz}$ for 1c) showed coupling with $\mathrm{C}_{\alpha}-\mathrm{H}(3.62 \mathrm{ppm}, J=8.6 \mathrm{~Hz}$ for 1c), $\mathrm{C} 4_{\beta}-\mathrm{H}\left(3.23 \mathrm{ppm}, J=8.6 \mathrm{~Hz}\right.$ for 1c) and with $\mathrm{C} 1^{\prime}-\mathrm{H}(2.58 \mathrm{ppm}, J=8.6,3.2 \mathrm{~Hz}$ for $1 \mathrm{c})$. However, in NOESY experiments $\mathrm{C} 5-\mathrm{H}$ hydrogen showed only one correlation with $\mathrm{C}_{\alpha}-\mathrm{H}$, which demonstrates the stereochemical anti relation between $\mathrm{C} 5-\mathrm{H}$ with $\mathrm{Cl}^{\prime}-\mathrm{H}$ and with $\mathrm{H} \mathrm{C}_{\beta}{ }^{-}$ H. Similar correlations were observed for the rest of the 1,3-oxazolidin-2-ones $\mathbf{1}$. Therefore, since the original asymmetric center in $\alpha$-dibenzylamino ester $\mathbf{2}$ had an $S$ absolute configuration and had not been involved in any epimerization-promoting processes, the absolute configuration of compounds 1a-e was established as 1'S, $5 R$.

\section{Conclusions}

In this work a new methodology has been developed for the synthesis of enantiopure 5substituted 1,3-oxazolidin-2-ones with potential biological activity from non-racemic $\alpha$ dibenzylamino esters in moderate yields and high stereoselectivity. Magnesium chloride and zinc iodide were tested as catalysts in the addition of trimethylsilyl cyanide to $\alpha$-dibenzylamino 
aldehydes showing higher stereo selectivity with zinc iodide in comparison with magnesium chloride or other catalysts reported previously. ${ }^{11}$

\section{Experimental Section}

General Procedures. All reagents were purchased in the higher quality available and were used without further purification. The solvents used in column chromatography were obtained from commercial suppliers and used without further distillation. Infrared spectra (FTIR) were recorded on a Perkin Elmer FT-IR 1600 spectrophotometer. Nuclear magnetic resonance ${ }^{1} \mathrm{H}$ (at $200 \mathrm{MHz}$ ) and ${ }^{13} \mathrm{C}$ (at $50 \mathrm{MHz}$ ) spectra were recorded on a Varian Mercury $200 \mathrm{MHz}$ Spectrometer in $\mathrm{CDCl}_{3}$ with TMS as internal standard. Liquid chromatograms were obtained on an Agilent 1100 Series LC with a reverse phase ZORBAX s $\beta$-C18 column (5 mm, 3 x $150 \mathrm{~mm}$ ) and MSD Trap. Electrospray ionization mass spectra (ESI-MS) were obtained with an ion trap, and the intensities are reported as a percentage relative to the base peak after the corresponding $m / z$ value. HR-MS were obtained in an Agilent LCTOF (2006), a high resolution TOF analyzer with Windows XP based OS and APCI/ESI ionization. Melting points were obtained on an Electrothermal 88629 apparatus and are uncorrected. Optical rotations were determined using an Autopol III polarimeter.

\section{General procedure for the synthesis of $\alpha$-dibenzylamino alcohols 11}

To a solution of the $\alpha$-dibenzylamino ester 2 in dry THF was added $\mathrm{LiBH}_{4}$ (2.0 equiv.) at $25^{\circ} \mathrm{C}$ under argon atmosphere. Then, the reaction mixture was refluxed for $8 \mathrm{~h}$. A saturated $\mathrm{NH}_{4} \mathrm{Cl}$ solution was added and the mixture stirred for $30 \mathrm{~min}$ and then filtered. The liquid phase was extracted with $\mathrm{CH}_{2} \mathrm{Cl}_{2}(3 \times 20 \mathrm{~mL})$. The organic layer was separated and dried over $\mathrm{Na}_{2} \mathrm{SO}_{4}$, filtered, and the solvent was evaporated under reduced pressure to give a crude product that was purified by flash chromatography when was necessary.

(S)-2-Dibenzylamino-3-phenylpropan-1-ol (11a). White solid. 99\% yield. FTIR (KBr): 3426, 3026, 2958, 1601, $1029 \mathrm{~cm}^{-1} .{ }^{1} \mathrm{H}$ NMR $\left(\mathrm{CDCl}_{3}, 200 \mathrm{MHz}\right): \delta 7.28(\mathrm{~m}, 15 \mathrm{H}), 4.64$ (br s, $\left.1 \mathrm{H}\right)$, $3.91(\mathrm{~d}, J=13.2 \mathrm{~Hz}, 2 \mathrm{H}), 3.47(\mathrm{~d}, J=13.2 \mathrm{~Hz}, 2 \mathrm{H}), 3.32(\mathrm{dd}, J=10.6,4.5 \mathrm{~Hz}, 1 \mathrm{H}), 3.10(\mathrm{~m}$, $3 \mathrm{H}), 2.42(\mathrm{dd}, J=14.0,10.6 \mathrm{~Hz}, 1 \mathrm{H}) .{ }^{13} \mathrm{C} \mathrm{NMR}\left(\mathrm{CDCl}_{3}, 50 \mathrm{MHz}\right): \delta 138.8(\mathrm{C}), 138.7(2 \times \mathrm{C})$, $128.7(6 \times \mathrm{CH}), 128.2(6 \times \mathrm{CH}), 127.0(2 \times \mathrm{CH}), 125.9(\mathrm{CH}), 60.7(\mathrm{CH}), 60.1\left(\mathrm{CH}_{2}\right), 53.2(2 \times$ $\left.\mathrm{CH}_{2}\right), 31.7\left(\mathrm{CH}_{2}\right)$. ESI-MS m/z: 332 [M+H] $]^{+}$; MS/MS m/z (rel. int.): 240(75), 224(96), 181(100), 117(30). ESI-MS $m / z: 354[\mathrm{M}+\mathrm{Na}]^{+}$

(S)-2-Dibenzylaminopropan-1-ol (11b). Colorless oil. 94\% yield. FTIR (neat): 3421, 3060, 2929, 1601, 1365, $1035 \mathrm{~cm}^{-1} .{ }^{1} \mathrm{H}$ NMR $\left(\mathrm{CDCl}_{3}, 200 \mathrm{MHz}\right): \delta 7.30(\mathrm{~m}, 10 \mathrm{H}), 4.63$ (br s, $\left.1 \mathrm{H}\right)$, $3.81(\mathrm{~d}, J=13.4 \mathrm{~Hz}, 2 \mathrm{H}), 3.45(\mathrm{t}, J=10.6 \mathrm{~Hz}, 1 \mathrm{H}), 3.35(\mathrm{~d}, J=13.4 \mathrm{~Hz}, 2 \mathrm{H}), 3.34$ (dt, $J=10.6$, $5.6 \mathrm{~Hz}, 1 \mathrm{H}), 2.98(\mathrm{~m}, 1 \mathrm{H}), 0.97(\mathrm{~d}, J=6.6 \mathrm{~Hz}, 3 \mathrm{H}) .{ }^{13} \mathrm{C} \mathrm{NMR}\left(\mathrm{CDCl}_{3}, 50 \mathrm{MHz}\right): \delta 138.8(2 \times$ C), $128.7(4 \times \mathrm{CH}), 128.2(4 \times \mathrm{CH}), 126.9(2 \times \mathrm{CH}), 62.5(\mathrm{CH}), 56.0\left(\mathrm{CH}_{2}\right), 52.7\left(2 \times \mathrm{CH}_{2}\right), 8.6$ 
$\left(\mathrm{CH}_{3}\right)$. ESI-MS $m / z: 256[\mathrm{M}+\mathrm{H}]^{+}$; MS/MS m/z (rel. int.): 181(96), 164(35), 148(60), 91(100). ESI-MS $m / z: 278[\mathrm{M}+\mathrm{Na}]^{+}$.

(S)-2-Dibenzylamino-4-methylpentan-1-ol (11c). Colorless oil. 76\% yield. FTIR (neat): 3430, 3027, 2955, 1601, $13641068 \mathrm{~cm}^{-1} .{ }^{1} \mathrm{H}$ NMR (CDCl $\left.3,200 \mathrm{MHz}\right): \delta 7.27(\mathrm{~m}, 10 \mathrm{H}), 3.88(\mathrm{~d}, J=$ $13.2 \mathrm{~Hz}, 2 \mathrm{H}), 3.67$ (d, $J=13.2 \mathrm{~Hz}, 2 \mathrm{H}), 3.62(\mathrm{br} \mathrm{s}, 1 \mathrm{H}), 3.57$ (dd, $J=10.6,4.8 \mathrm{~Hz}, 1 \mathrm{H}), 3.43$ (dd, $J=10.6,9.6 \mathrm{~Hz}, 1 \mathrm{H}), 2.51$ (ddd, $J=9.6,8.6,4.8 \mathrm{~Hz}, 1 \mathrm{H}), 2.04(\mathrm{~m}, 1 \mathrm{H}), 1.13(\mathrm{~d}, J=6.6 \mathrm{~Hz}$, $3 \mathrm{H}), 0.88(\mathrm{~d}, J=6.6 \mathrm{~Hz}, 3 \mathrm{H}) .{ }^{13} \mathrm{C} \mathrm{NMR}\left(\mathrm{CDCl}_{3}, 50 \mathrm{MHz}\right): \delta 139.7(2 \times \mathrm{C}), 128.8(4 \times \mathrm{CH})$, $128.4(4 \times \mathrm{CH}), 127.2(2 \times \mathrm{CH}), 64.7(\mathrm{CH}), 59.2\left(\mathrm{CH}_{2}\right), 54.2\left(2 \times \mathrm{CH}_{2}\right), 27.6(\mathrm{CH}), 22.7\left(\mathrm{CH}_{3}\right)$, $22.1\left(\mathrm{CH}_{3}\right)$. ESI-MS $\mathrm{m} / z: 284[\mathrm{M}+\mathrm{H}]^{+} ; \mathrm{MS} / \mathrm{MS} \mathrm{m} / z$ (rel. int.): 192(35), 181(90), 120(90), 91(100). ESI-MS $m / z: 306[\mathrm{M}+\mathrm{Na}]^{+}$.

(S)-2-Dibenzylamino-3-methylbutan-1-ol (11d). Colorless oil. 60\% yield. FTIR (neat): 3440, 3060, 2952, 1600, 1151, $1030 \mathrm{~cm}^{-1} .{ }^{1} \mathrm{H}$ NMR $\left(\mathrm{CDCl}_{3}, 200 \mathrm{MHz}\right): \delta 7.27(\mathrm{~m}, 10 \mathrm{H}), 3.80(\mathrm{~d}, J=$ $13.2 \mathrm{~Hz}, 2 \mathrm{H}), 3.44(\mathrm{~m}, 2 \mathrm{H}), 3.36(\mathrm{~d}, J=13.2 \mathrm{~Hz}, 2 \mathrm{H}), 3.25(\mathrm{br} \mathrm{s}, 1 \mathrm{H}), 2.84(\mathrm{~m}, 1 \mathrm{H}), 1.51(\mathrm{~m}$, $2 \mathrm{H}), 1.15(\mathrm{~m}, 1 \mathrm{H}), 0.91(\mathrm{~d}, J=6.2 \mathrm{~Hz}, 3 \mathrm{H}), 0.85(\mathrm{~d}, J=6.4 \mathrm{~Hz}, 3 \mathrm{H}) .{ }^{13} \mathrm{C} \mathrm{NMR}\left(\mathrm{CDCl}_{3}, 50\right.$ $\mathrm{MHz}): \delta 139.0(2 \times \mathrm{C}), 128.7(4 \times \mathrm{CH}), 128.1(4 \times \mathrm{CH}), 126.9(2 \times \mathrm{CH}), 67.7(\mathrm{CH}), 56.7\left(\mathrm{CH}_{2}\right)$, $52.9\left(2 \times \mathrm{CH}_{2}\right), 33.9\left(\mathrm{CH}_{2}\right), 25.4(\mathrm{CH}), 23.9\left(\mathrm{CH}_{3}\right), 22.0\left(\mathrm{CH}_{3}\right)$. ESI-MS m/z: $298[\mathrm{M}+\mathrm{H}]^{+}$; MS/MS $m / z$ (rel. int.): 206(50), 190(96), 181(90), 91(100). ESI-MS $m / z: 320[\mathrm{M}+\mathrm{Na}]^{+}$.

(2S,3S)-2-Dibenzylamino-3-methylpentan-1-ol (11e). Colorless oil. 44\% yield. FTIR (neat): 3376, 3060, 2962, 1601, 1377, 1070, $1027 \mathrm{~cm}^{-1} .{ }^{1} \mathrm{H} \mathrm{NMR}\left(\mathrm{CDCl}_{3}, 200 \mathrm{MHz}\right): \delta 7.26(\mathrm{~m}, 10 \mathrm{H})$, 4.67 (br s, 1H), 3.88 (d, $J=13.2 \mathrm{~Hz}, 2 \mathrm{H}), 3.59$ (d, $J=13.2 \mathrm{~Hz}, 2 \mathrm{H}), 3.53$ (d, $J=7.2 \mathrm{~Hz}, 2 \mathrm{H})$, $2.64(\mathrm{q}, J=7.0 \mathrm{~Hz}, 1 \mathrm{H}), 1.87(\mathrm{~m}, 1 \mathrm{H}), 1.60(\mathrm{~m}, 1 \mathrm{H}), 1.25(\mathrm{~m}, 1 \mathrm{H}), 0.90(\mathrm{~d}, J=4.4 \mathrm{~Hz}, 3 \mathrm{H}), 0.87$ $(\mathrm{t}, J=6.6 \mathrm{~Hz}, 3 \mathrm{H}) \cdot{ }^{13} \mathrm{C} \mathrm{NMR}\left(\mathrm{CDCl}_{3}, 50 \mathrm{MHz}\right): \delta 139.4(2 \times \mathrm{C}), 128.8(4 \times \mathrm{CH}), 128.1(4 \times$ $\mathrm{CH}), 126.8(2 \times \mathrm{CH}), 62.7(\mathrm{CH}), 58.6\left(\mathrm{CH}_{2}\right), 53.9\left(2 \times \mathrm{CH}_{2}\right), 32.7(\mathrm{CH}), 28.3\left(\mathrm{CH}_{2}\right), 15.9\left(\mathrm{CH}_{3}\right)$, $11.5\left(\mathrm{CH}_{3}\right)$. ESI-MS m/z: $298[\mathrm{M}+\mathrm{H}]^{+} ; \mathrm{MS} / \mathrm{MS} \mathrm{m} / \mathrm{z}$ (rel. int.): 206(35), 181(95), 120(85), 91(100). ESI-MS $m / z: 320[\mathrm{M}+\mathrm{Na}]^{+}$.

\section{General procedure for the synthesis of $\alpha$-dibenzylamino aldehydes 3}

To a solution of oxalyl chloride (1.1 equiv.) in dry $\mathrm{CH}_{2} \mathrm{Cl}_{2}$ was added dropwise a solution of DMSO (2.2 equiv.) in dry $\mathrm{CH}_{2} \mathrm{Cl}_{2}$ at $-78{ }^{\circ} \mathrm{C}$ under argon atmosphere and the mixture was stirred for $10 \mathrm{~min}$. Then, a solution of the dibenzylamino alcohol $\mathbf{1 1}$ in dry $\mathrm{CH}_{2} \mathrm{Cl}_{2}$ was added dropwise and the reaction mixture was stirred for $30 \mathrm{~min}$ at the same temperature. After this time, a solution of triethylamine (5 equiv.) in $\mathrm{CH}_{2} \mathrm{Cl}_{2}$ was added dropwise and the mixture was stirred for $1 \mathrm{~h}$ and allowed to reach room temperature. The organic layer was washed with brine, separated, dried over $\mathrm{Na}_{2} \mathrm{SO}_{4}$, and filtered. The solvent was evaporated under reduced pressure to give a crude product that was used in the next reaction without further purification.

(S)-2-Dibenzylamino-3-phenylpropanal (3a). Yellow oil. 95\% yield. FTIR (neat): 3026, 2922, 2709, 1727, $1122 \mathrm{~cm}^{-1} .{ }^{1} \mathrm{H}$ NMR $\left(\mathrm{CDCl}_{3}, 200 \mathrm{MHz}\right): \delta 9.70(\mathrm{~s}, 1 \mathrm{H}), 7.27(\mathrm{~m}, 15 \mathrm{H}), 3.82(\mathrm{~d}, J=$ $13.6 \mathrm{~Hz}, 2 \mathrm{H}), 3.66(\mathrm{~d}, J=13.6 \mathrm{~Hz}, 2 \mathrm{H}), 3.55(\mathrm{t}, J=6.8 \mathrm{~Hz}, 1 \mathrm{H}), 3.14(\mathrm{dd}, J=13.6,7.2 \mathrm{~Hz}, 1 \mathrm{H})$, $2.92(\mathrm{dd}, J=13.6,6.2 \mathrm{~Hz}, 1 \mathrm{H}) .{ }^{13} \mathrm{C}$ NMR $\left(\mathrm{CDCl}_{3}, 50 \mathrm{MHz}\right): \delta 201.7(\mathrm{CH}), 138.8(\mathrm{C}), 138.5(2 \times$ 
C), $129.1(2 \times \mathrm{CH}), 128.4(4 \times \mathrm{C}), 128.1(6 \times \mathrm{CH}), 127.0(2 \times \mathrm{CH}), 125.9(\mathrm{CH}), 68.3(\mathrm{CH}), 54.7$ $\left(2 \times \mathrm{CH}_{2}\right), 30.0\left(\mathrm{CH}_{2}\right)$. ESI-MS m/z: $362[\mathrm{M}+\mathrm{MeOH}+\mathrm{H}]^{+} ; \mathrm{MS} / \mathrm{MS} m / z$ (rel. int.): 344(100), $330(35)$.

(S)-2-Dibenzylaminopropanal (3b). Yellow oil. 83\% yield. FTIR (neat): 3060, 2807, 2706, 1725, 1372, 1149, $1028 \mathrm{~cm}^{-1} .{ }^{1} \mathrm{H}$ NMR $\left(\mathrm{CDCl}_{3}, 200 \mathrm{MHz}\right): \delta 9.71$ (s, 1H), $7.28(\mathrm{~m}, 10 \mathrm{H}), 3.73$ $(\mathrm{d}, J=13.6 \mathrm{~Hz}, 2 \mathrm{H}), 3.55(\mathrm{~d}, J=13.6 \mathrm{~Hz}, 2 \mathrm{H}), 2.77(\mathrm{q}, J=6.6 \mathrm{~Hz}, 1 \mathrm{H}), 1.17(\mathrm{~d}, J=6.6 \mathrm{~Hz}, 3 \mathrm{H})$. ${ }^{13} \mathrm{C} \mathrm{NMR}\left(\mathrm{CDCl}_{3}, 50 \mathrm{MHz}\right): \delta 204.0(\mathrm{CH}), 138.7(2 \times \mathrm{C}), 128.5(4 \times \mathrm{CH}), 128.1(4 \times \mathrm{CH}), 127.0$ $(2 \times \mathrm{CH}), 62.7(\mathrm{CH}), 54.8\left(2 \times \mathrm{CH}_{2}\right), 6.7\left(\mathrm{CH}_{3}\right)$. ESI-MS m/z: $254[\mathrm{M}+\mathrm{H}]^{+}$.

(S)-2-Dibenzylamino-3-methylbutanal (3c). Yellow oil. 97\% yield. FTIR (neat): 3060, 2961, 2723, 1728, $1198 \mathrm{~cm}^{-1} .{ }^{1} \mathrm{H}$ NMR $\left(\mathrm{CDCl}_{3}, 200 \mathrm{MHz}\right): \delta 9.83(\mathrm{~d}, J=3.4 \mathrm{~Hz}, 1 \mathrm{H}), 7.32(\mathrm{~m}, 10 \mathrm{H})$, $4.01(\mathrm{~d}, J=13.6 \mathrm{~Hz}, 2 \mathrm{H}), 3.70(\mathrm{~d}, J=13.6 \mathrm{~Hz}, 2 \mathrm{H}), 2.71(\mathrm{dd}, J=10.0,3.4 \mathrm{~Hz}, 1 \mathrm{H}), 2.25(\mathrm{~m}$, $1 \mathrm{H}), 1.07(\mathrm{~d}, J=6.6 \mathrm{~Hz}, 3 \mathrm{H}), 0.85(\mathrm{~d}, J=6.6 \mathrm{~Hz}, 3 \mathrm{H}) .{ }^{13} \mathrm{C} \mathrm{NMR}\left(\mathrm{CDCl}_{3}, 50 \mathrm{MHz}\right)$ : $\delta 204.6(\mathrm{CH}), 138.8(2 \times \mathrm{C}), 128.4(4 \times \mathrm{CH}), 128.0(4 \times \mathrm{CH}), 126.8(2 \times \mathrm{CH}), 71.3(\mathrm{CH}), 54.4(2$ $\left.\times \mathrm{CH}_{2}\right), 25.9(\mathrm{CH}), 20.1\left(\mathrm{CH}_{3}\right), 19.7\left(\mathrm{CH}_{3}\right)$. ESI-MS m/z: $282[\mathrm{M}+\mathrm{H}]^{+}$. ESI-MS m/z: 314 $[\mathrm{M}+\mathrm{MeOH}+\mathrm{H}]^{+}$.

(S)-2-Dibenzylamino-4-methylpentanal (3d). Yellow oil. 98\% yield. FTIR (neat): 3061, 2954, 2706, 1727, 1370, $1145 \mathrm{~cm}^{-1} .{ }^{1} \mathrm{H}$ NMR $\left(\mathrm{CDCl}_{3}, 200 \mathrm{MHz}\right): \delta 9.74(\mathrm{~s}, 1 \mathrm{H}), 7.31(\mathrm{~m}, 10 \mathrm{H}), 3.78$ $(\mathrm{d}, J=13.8 \mathrm{~Hz}, 2 \mathrm{H}), 3.69(\mathrm{~d}, J=13.8 \mathrm{~Hz}, 2 \mathrm{H}), 3.21(\mathrm{t}, J=6.4 \mathrm{~Hz}, 1 \mathrm{H}), 1.56(\mathrm{~m}, 3 \mathrm{H}), 0.82(\mathrm{~d}, J$ $=6.6 \mathrm{~Hz}, 3 \mathrm{H}), 0.77(\mathrm{~d}, J=6.6 \mathrm{~Hz}, 3 \mathrm{H}) .{ }^{13} \mathrm{C} \mathrm{NMR}\left(\mathrm{CDCl}_{3}, 50 \mathrm{MHz}\right): \delta 203.5(\mathrm{CH}), 138.9(2 \times$ C), $128.5(4 \times \mathrm{CH}), 128.0(4 \times \mathrm{CH}), 126.9(2 \times \mathrm{CH}), 64.6(\mathrm{CH}), 54.6\left(2 \times \mathrm{CH}_{2}\right), 32.9\left(\mathrm{CH}_{2}\right)$, $25.1(\mathrm{CH}), 22.6\left(\mathrm{CH}_{3}\right), 22.4 \quad\left(\mathrm{CH}_{3}\right)$. ESI-MS m/z: $296 \quad[\mathrm{M}+\mathrm{H}]^{+}$. ESI-MS $m / z: 328$ $[\mathrm{M}+\mathrm{MeOH}+\mathrm{H}]^{+}$.

(2S,3S)-2-Dibenzylamino-3-methylpentanal (3e). Yellow oil. 96\% yield. FTIR (neat): 3030, 2964, 2730, 1723, 1207, $1028 \mathrm{~cm}^{-1} .{ }^{1} \mathrm{H} \mathrm{NMR}\left(\mathrm{CDCl}_{3}, 200 \mathrm{MHz}\right): \delta 9.84(\mathrm{~d}, J=4.0 \mathrm{~Hz}, 1 \mathrm{H})$, $7.31(\mathrm{~m}, 10 \mathrm{H}), 4.00(\mathrm{~d}, J=13.7 \mathrm{~Hz}, 2 \mathrm{H}), 3.68(\mathrm{~d}, J=13.7 \mathrm{~Hz}, 2 \mathrm{H}), 2.81(\mathrm{dd}, J=9.6,4.0 \mathrm{~Hz}$, $1 \mathrm{H}), 2.07(\mathrm{~m}, 1 \mathrm{H}), 1.87(\mathrm{~m}, 1 \mathrm{H}), 1.19(\mathrm{~m}, 1 \mathrm{H}), 0.84(\mathrm{~d}, J=6.6 \mathrm{~Hz}, 3 \mathrm{H}), 0.79(\mathrm{t}, J=7.4 \mathrm{~Hz}, 3 \mathrm{H})$. ${ }^{13} \mathrm{C} \mathrm{NMR}\left(\mathrm{CDCl}_{3}, 50 \mathrm{MHz}\right): \delta 205.0(\mathrm{CH}), 138.8(2 \times \mathrm{C}), 128.5(4 \times \mathrm{CH}), 128.0(4 \times \mathrm{CH}), 126.8$ $(2 \times \mathrm{CH}), 69.8(\mathrm{CH}), 54.4\left(2 \times \mathrm{CH}_{2}\right), 31.9(\mathrm{CH}), 25.4\left(\mathrm{CH}_{2}\right), 15.8\left(\mathrm{CH}_{3}\right), 10.4\left(\mathrm{CH}_{3}\right)$. ESI-MS $m / z: 296[\mathrm{M}+\mathrm{H}]^{+} ; \mathrm{MS} / \mathrm{MS} m / z$ (rel. int.): 206(35), 181(95), 120(85), 91(100). ESI-MS m/z: 328 $[\mathrm{M}+\mathrm{MeOH}+\mathrm{H}]^{+}$.

\section{General procedure for the synthesis of trimethylsilylcyanohydrins 12}

To a solution of aldehyde 3 (1 .0 equiv.) in dry $\mathrm{CH}_{2} \mathrm{Cl}_{2}$ was added $\mathrm{ZnI}_{2}$ (1.0 equiv.) at $0{ }^{\circ} \mathrm{C}$ under argon atmosphere and the mixture was stirred for $10 \mathrm{~min}$. Then, trimethylsilyl cyanide (1.0 equiv.) was added dropwise and the reaction mixture was stirred for $2 \mathrm{~h}$ at the same temperature. After this time, water was added and the mixture was stirred for $5 \mathrm{~min}$ and allowed to reach room temperature. The organic layer was washed with brine, separated, dried over $\mathrm{Na}_{2} \mathrm{SO}_{4}$, and filtered. The solvent was evaporated under reduced pressure to give a pure crude product that was used in the next reaction without further purification. 
(2S,3S)-3-Dibenzylamino-4-phenyl-2-(trimethylsilyloxy)butanenitrile (12a). Colorless oil. 99\% yield. FTIR (neat): 3028, 2950, 2368, 1254, 1114, $850 \mathrm{~cm}^{-1} .{ }^{1} \mathrm{H} \mathrm{NMR}\left(\mathrm{CDCl}_{3}, 200 \mathrm{MHz}\right): \delta$ $7.22(\mathrm{~m}, 15 \mathrm{H}), 4.46(\mathrm{~d}, J=6.2 \mathrm{~Hz}, 1 \mathrm{H}), 3.72(\mathrm{br} \mathrm{s}, 4 \mathrm{H}), 3.38(\mathrm{dt}, J=8.0,6.2 \mathrm{~Hz}, 1 \mathrm{H}), 2.99(\mathrm{~m}$, 2H), $0.17(\mathrm{~s}, 9 \mathrm{H}) .{ }^{13} \mathrm{C} \mathrm{NMR}\left(\mathrm{CDCl}_{3}, 50 \mathrm{MHz}\right): \delta 138.9(\mathrm{C}), 138.6(2 \times \mathrm{C}), 129.1(2 \times \mathrm{CH}), 128.5$ $(4 \times \mathrm{CH}), 128.0(2 \times \mathrm{CH}), 127.9(4 \times \mathrm{CH}), 126.8(2 \times \mathrm{CH}), 126.0(\mathrm{CH}), 119.5(\mathrm{C}), 63.0(\mathrm{CH})$, $62.7(\mathrm{CH}), 54.7\left(2 \times \mathrm{CH}_{2}\right), 33.1\left(\mathrm{CH}_{2}\right),-0.4\left(3 \times \mathrm{CH}_{3}\right)$. ESI-MS $m / z: 429[\mathrm{M}+\mathrm{H}]^{+} ; \mathrm{MS} / \mathrm{MS} m / z$ (rel. int.): 311(100), 210(20). ESI-MS $m / z: 451[\mathrm{M}+\mathrm{Na}]^{+}$.

(2S,3S)-3-Dibenzylamino-2-(trimethylsilyloxy)butanenitrile (12b). Colorless oil. 97\% yield. FTIR (neat): 3061, 2959, 2212, 1253, 1092, $845 \mathrm{~cm}^{-1} .{ }^{1} \mathrm{H}$ NMR $\left(\mathrm{CDCl}_{3}, 200 \mathrm{MHz}\right): \delta 7.43(\mathrm{~m}$, $10 \mathrm{H}), 4.47(\mathrm{~d}, J=8.8 \mathrm{~Hz}, 1 \mathrm{H}), 3.87(\mathrm{~d}, J=13.6 \mathrm{~Hz}, 2 \mathrm{H}), 3.64(\mathrm{~d}, J=13.6 \mathrm{~Hz}, 2 \mathrm{H}), 3.24(\mathrm{~m}$, $1 \mathrm{H}), 1.24(\mathrm{~d}, J=6.6 \mathrm{~Hz}, 3 \mathrm{H}), 0.14(\mathrm{~s}, 9 \mathrm{H}) \cdot{ }^{13} \mathrm{C} \mathrm{NMR}\left(\mathrm{CDCl}_{3}, 50 \mathrm{MHz}\right): \delta 139.0(2 \times \mathrm{C}), 128.8$ $(4 \times \mathrm{CH}), 128.4(4 \times \mathrm{CH}), 127.2(2 \times \mathrm{CH}), 120.0(\mathrm{C}), 65.2(\mathrm{CH}), 57.6(\mathrm{CH}), 55.1\left(2 \times \mathrm{CH}_{2}\right), 9.5$ $\left(\mathrm{CH}_{3}\right), 0.08\left(3 \times \mathrm{CH}_{3}\right)$. ESI-MS $m / z: 353[\mathrm{M}+\mathrm{H}]^{+}$. ESI-MS $m / z: 375[\mathrm{M}+\mathrm{Na}]^{+}$.

(2S,3S)-3-Dibenzylamino-4-methyl-2-(trimethylsilyloxy)pentanenitrile (12c). Colorless oil. $82 \%$ yield. FTIR (neat): 3062, 2957, 2218, 1250, 1085, $840 \mathrm{~cm}^{-1} .{ }^{1} \mathrm{H}$ NMR $\left(\mathrm{CDCl}_{3}, 200 \mathrm{MHz}\right): \delta$ $7.32(\mathrm{~m}, 10 \mathrm{H}), 4.77(\mathrm{~d}, J=4.4 \mathrm{~Hz}, 1 \mathrm{H}) 4.19(\mathrm{~d}, J=13.2 \mathrm{~Hz}, 2 \mathrm{H}), 3.73(\mathrm{~d}, J=13.2 \mathrm{~Hz}, 2 \mathrm{H}), 2.66$ $(\mathrm{dd}, J=10.6,5.8 \mathrm{~Hz}, 1 \mathrm{H}), 2.15(\mathrm{~m}, 1 \mathrm{H}), 1.02(\mathrm{~d}, J=6.6 \mathrm{~Hz}, 3 \mathrm{H}), 0.97(\mathrm{~d}, J=6.6 \mathrm{~Hz}, 3 \mathrm{H}), 0.27$ $(\mathrm{s}, 9 \mathrm{H}) \cdot{ }^{13} \mathrm{C} \mathrm{NMR}\left(\mathrm{CDCl}_{3}, 50 \mathrm{MHz}\right): \delta 138.9(2 \times \mathrm{C}), 129.2(4 \times \mathrm{CH}), 128.5(4 \times \mathrm{CH}), 127.4(2$ $\times \mathrm{CH}), 119.9(\mathrm{C}), 64.6(\mathrm{CH}), 59.3(\mathrm{CH}), 54.6\left(2 \times \mathrm{CH}_{2}\right), 28.9(\mathrm{CH}), 22.4\left(\mathrm{CH}_{3}\right), 20.1\left(\mathrm{CH}_{3}\right),-0.2$ $\left(3 \times \mathrm{CH}_{3}\right)$. ESI-MS m/z: 367 [M-Me+2H] ${ }^{+}$; MS/MS m/z (rel. int.): 325(95), 275(100), 199(10).

(2S,3S)-3-Dibenzylamino-5-methyl-2-(trimethylsilyloxy)hexanenitrile (12d). Colorless oil. 94\% yield. FTIR (neat): 3061, 2955, 2228, 1255, 1106, $848 \mathrm{~cm}^{-1} .{ }^{1} \mathrm{H} \mathrm{NMR}\left(\mathrm{CDCl}_{3}, 200 \mathrm{MHz}\right): \delta$ $7.34(\mathrm{~m}, 10 \mathrm{H}), 4.45(\mathrm{~d}, J=5.8 \mathrm{~Hz}, 1 \mathrm{H}), 3.81(\mathrm{~d}, J=13.8 \mathrm{~Hz}, 2 \mathrm{H}), 3.63(\mathrm{~d}, J=13.8 \mathrm{~Hz}, 2 \mathrm{H})$, $3.00(\mathrm{~m}, 1 \mathrm{H}), 1.75(\mathrm{~m}, 2 \mathrm{H}), 1.25(\mathrm{~m}, 1 \mathrm{H}), 0.90(\mathrm{~d}, J=6.6 \mathrm{~Hz}, 3 \mathrm{H}), 0.70(\mathrm{~d}, J=6.6 \mathrm{~Hz}, 3 \mathrm{H}), 0.20$ $(\mathrm{s}, 9 \mathrm{H}) \cdot{ }^{13} \mathrm{C} \mathrm{NMR}\left(\mathrm{CDCl}_{3}, 50 \mathrm{MHz}\right): \delta 139.0(2 \times \mathrm{C}), 128.6(4 \times \mathrm{CH}), 128.0(4 \times \mathrm{CH}), 126.8(2$ $\times \mathrm{CH}), 119.8(\mathrm{C}), 63.2(\mathrm{CH}), 58.8(\mathrm{CH}), 54.6\left(2 \times \mathrm{CH}_{2}\right), 36.4\left(\mathrm{CH}_{2}\right), 25.0(\mathrm{CH}), 23.3\left(\mathrm{CH}_{3}\right)$, $22.0\left(\mathrm{CH}_{3}\right),-0.4\left(3 \times \mathrm{CH}_{3}\right)$. ESI-MS $m / z: 395[\mathrm{M}+\mathrm{H}]^{+}$. ESI-MS $m / z: 417[\mathrm{M}+\mathrm{Na}]^{+}$. ESI-MS $m / z$ : $381[\mathrm{M}-\mathrm{Me}+2 \mathrm{H}]^{+}$; MS/MS m/z (rel. int.): 290(100), 248(10), 213(12), 157(10).

(2S,3S,4S)-3-Dibenzylamino-4-methyl-2-(trimethylsilyloxy)hexanenitrile (12e). Colorless oil. $72 \%$ yield. FTIR (neat): 3060, 2958, 2232, 1245, 1102, $845 \mathrm{~cm}^{-1} .{ }^{1} \mathrm{H}$ NMR $\left(\mathrm{CDCl}_{3}, 200 \mathrm{MHz}\right): \delta$ $7.33(\mathrm{~m}, 10 \mathrm{H}), 4.78(\mathrm{~d}, J=4.8 \mathrm{~Hz}, 1 \mathrm{H}), 3.87(\mathrm{~d}, J=13.6 \mathrm{~Hz}, 2 \mathrm{H}), 3.65(\mathrm{~d}, J=13.6 \mathrm{~Hz}, 2 \mathrm{H})$, $2.79(\mathrm{dd}, J=7.8,4.8 \mathrm{~Hz}, 1 \mathrm{H}), 1.94(\mathrm{~m}, 2 \mathrm{H}), 1.17(\mathrm{~m}, 1 \mathrm{H}), 0.97(\mathrm{~d}, J=7.0 \mathrm{~Hz}, 3 \mathrm{H}), 0.75(\mathrm{t}, J=$ $7.4 \mathrm{~Hz}, 3 \mathrm{H}), 0.24(\mathrm{~s}, 9 \mathrm{H}) .{ }^{13} \mathrm{C} \mathrm{NMR}\left(\mathrm{CDCl}_{3}, 50 \mathrm{MHz}\right): \delta 139.1(2 \times \mathrm{C}), 129.0(4 \times \mathrm{CH}), 128.2(4$ $\times \mathrm{CH}), 126.9(2 \times \mathrm{CH}), 120.3(\mathrm{C}), 64.0(\mathrm{CH}), 61.0(\mathrm{CH}), 54.9\left(2 \times \mathrm{CH}_{2}\right), 33.0(\mathrm{CH}), 27.1\left(\mathrm{CH}_{2}\right)$, $16.0\left(\mathrm{CH}_{3}\right), 11.2\left(\mathrm{CH}_{3}\right),-0.2\left(3 \times \mathrm{CH}_{3}\right)$. ESI-MS m/z: $395[\mathrm{M}+\mathrm{H}]^{+}$. ESI-MS m/z: $417[\mathrm{M}+\mathrm{Na}]^{+}$.

\section{General procedure for the synthesis of $\alpha$-dibenzylamino amino alcohols 13}

To a solution of trimethylsilylcyanohydrins 12 (1.0 equiv.) in dry THF was added drop wise a solution of $\mathrm{LiAlH}_{4}$ (2.0 equiv.) in dry diethyl ether at $0{ }^{\circ} \mathrm{C}$ under argon atmosphere and stirred for $5 \mathrm{~h}$ at same temperature. Then, a $5 \% \mathrm{KOH}$ solution was added drop wise until a white solid was 
form and the reaction mixture was filtered. The organic layer was dried over $\mathrm{Na}_{2} \mathrm{SO}_{4}$, filtered and the solvent was evaporated under reduced pressure to give a crude product that was used in the next reaction without further purification.

(2R,3S)-1-Amino-3-dibenzylamino-4-phenylbutan-2-ol (13a). Colorless oil. 94\% yield. FTIR (neat): 3365, 3299, 2926, 1600, 1251, $1110 \mathrm{~cm}^{-1} .{ }^{1} \mathrm{H} \mathrm{NMR}\left(\mathrm{CDCl}_{3}, 200 \mathrm{MHz}\right): \delta 7.21$ (m, 15H), $4.63(\mathrm{~s}, 1 \mathrm{H}), 3.93(\mathrm{dd}, J=13.4,9.4 \mathrm{~Hz}, 1 \mathrm{H}), 3.70(\mathrm{~d}, J=13.6 \mathrm{~Hz}, 2 \mathrm{H}), 3.57(\mathrm{~d}, J=13.6 \mathrm{~Hz}, 2 \mathrm{H})$, $3.43(\mathrm{dd}, J=15.0,13.4 \mathrm{~Hz}, 1 \mathrm{H}), 2.82(\mathrm{~m}, 4 \mathrm{H}), 1.96($ br s, $2 \mathrm{H}) .{ }^{13} \mathrm{C} \mathrm{NMR}\left(\mathrm{CDCl}_{3}, 50 \mathrm{MHz}\right): \delta$ $141.1(\mathrm{C}), 139.5(2 \times \mathrm{C}), 129.1(2 \times \mathrm{CH}), 128.5(4 \times \mathrm{CH}), 128.2(2 \times \mathrm{CH}), 127.9(4 \times \mathrm{CH}), 126.6$ $(2 \times \mathrm{CH}), 125.5(\mathrm{CH}), 72.0(\mathrm{CH}), 61.4(\mathrm{CH}), 54.4\left(2 \times \mathrm{CH}_{2}\right), 44.6\left(\mathrm{CH}_{2}\right), 32.5\left(\mathrm{CH}_{2}\right)$. ESI-MS $m / z: 361[\mathrm{M}+\mathrm{H}]^{+} ; \mathrm{MS} / \mathrm{MS} \mathrm{m} / z$ (rel. int.): 344(98), 300(20), 210 (100).

(2R,3S)-1-Amino-3-dibenzylaminobutan-2-ol (13b). Colorless oil. 81\% yield. FTIR (neat): 3363, 3284, 3027, 2958, 1578, $1076 \mathrm{~cm}^{-1} .{ }^{1} \mathrm{H} \mathrm{NMR}\left(\mathrm{CDCl}_{3}, 200 \mathrm{MHz}\right): \delta 7.25(\mathrm{~m}, 10 \mathrm{H}), 4.59(\mathrm{~s}$, $1 \mathrm{H}), 3.65$ (d, $J=13.6 \mathrm{~Hz}, 2 \mathrm{H}), 3.62$ (dd, $J=11.0,5.8 \mathrm{~Hz}, 1 \mathrm{H}), 3.43(\mathrm{~m}, 1 \mathrm{H}), 3.31$ (d, $J=13.6$ $\mathrm{Hz}, 2 \mathrm{H}), 2.76(\mathrm{dd}, J=11.0,6.4 \mathrm{~Hz}, 1 \mathrm{H}), 2.55$ (q, $J=6.6 \mathrm{~Hz}, 1 \mathrm{H}), 1.76(\mathrm{br} \mathrm{s}, 2 \mathrm{H}), 1.10(\mathrm{~d}, J=$ $6.6 \mathrm{~Hz}, 3 \mathrm{H}) .{ }^{13} \mathrm{C} \mathrm{NMR}\left(\mathrm{CDCl}_{3}, 50 \mathrm{MHz}\right): \delta 140.3(2 \times \mathrm{C}), 129.1(4 \times \mathrm{CH}), 128.5(4 \times \mathrm{CH})$, $127.2(2 \times \mathrm{CH}), 73.5(\mathrm{CH}), 65.6(\mathrm{CH}), 54.7\left(2 \times \mathrm{CH}_{2}\right), 44.6\left(\mathrm{CH}_{2}\right), 12.1\left(\mathrm{CH}_{3}\right)$. ESI-MS m/z: 286 $[\mathrm{M}+\mathrm{H}]^{+} ; \mathrm{MS} / \mathrm{MS} m / z$ (rel. int.): 268(100), 199(5), 91(10).

(2R,3S)-1-Amino-3-dibenzylamino-5-methylhexan-2-ol (13c). Colorless oil. 92\% yield. FTIR (neat): 3342, 3283, 3061, 2955, 1601, 1361, $1068 \mathrm{~cm}^{-1} .{ }^{1} \mathrm{H} \mathrm{NMR}\left(\mathrm{CDCl}_{3}, 200 \mathrm{MHz}\right): \delta 7.24(\mathrm{~m}$, $10 \mathrm{H}), 3.87$ (d, $J=13.2 \mathrm{~Hz}, 2 \mathrm{H}), 3.76(\mathrm{~s}, 1 \mathrm{H}), 3.66(\mathrm{~d}, J=13.2 \mathrm{~Hz}, 2 \mathrm{H}), 3.56(\mathrm{dd}, J=10.6,4.8$ $\mathrm{Hz}, 1 \mathrm{H}), 3.42$ (t, $J=10.6 \mathrm{~Hz}, 1 \mathrm{H}), 3.19(\mathrm{br} \mathrm{s}, 2 \mathrm{H}), 2.52(\mathrm{~m}, 1 \mathrm{H}), 2.06(\mathrm{~m}, 1 \mathrm{H}), 1.12(\mathrm{~d}, J=6.6$ $\mathrm{Hz}, 3 \mathrm{H}), 0.87(\mathrm{~d}, J=7.0 \mathrm{~Hz}, 3 \mathrm{H}) .{ }^{13} \mathrm{C} \mathrm{NMR}\left(\mathrm{CDCl}_{3}, 50 \mathrm{MHz}\right): \delta 139.5(2 \times \mathrm{C}), 128.9(4 \times \mathrm{CH})$, $128.0(4 \times \mathrm{CH}), 126.8(2 \times \mathrm{CH}), 70.5(\mathrm{CH}), 64.0(\mathrm{CH}), 55.1\left(2 \times \mathrm{CH}_{2}\right), 44.7\left(\mathrm{CH}_{2}\right), 26.3(\mathrm{CH})$, $23.6\left(\mathrm{CH}_{3}\right), 20.1\left(\mathrm{CH}_{3}\right)$. ESI-MS $m / z: 313[\mathrm{M}+\mathrm{H}]^{+}$; MS/MS $m / z$ (rel. int.): 296(100), 278(5), 199(10), 91(7).

(2R,3S)-1-Amino-3-dibenzylamino-5-methylhexan-2-ol (13d). Colorless oil. 89\% yield. FTIR (neat): 3300, 3061, 2952, 1601, 1366, $1072 \mathrm{~cm}^{-1} .{ }^{1} \mathrm{H}$ NMR $\left(\mathrm{CDCl}_{3}, 200 \mathrm{MHz}\right): \delta 7.30(\mathrm{~m}, 10 \mathrm{H})$, $3.76(\mathrm{~m}, 1 \mathrm{H}), 3.63(\mathrm{~s}, 1 \mathrm{H}), 3.62(\mathrm{br} \mathrm{s}, 4 \mathrm{H}), 3.38(\mathrm{dd}, J=13.2,11.8 \mathrm{~Hz}, 1 \mathrm{H}), 2.76(\mathrm{dd}, J=12.6$, $3.8 \mathrm{~Hz}, 1 \mathrm{H}) 2.66(\mathrm{~m}, 3 \mathrm{H}), 1.60(\mathrm{~m}, 1 \mathrm{H}), 1.83$ (br s, 2H), 0.91 (d, $J=6.6 \mathrm{~Hz}, 3 \mathrm{H}), 0.73(\mathrm{~d}, J=6.4$ $\mathrm{Hz}, 3 \mathrm{H}) .{ }^{13} \mathrm{C} \mathrm{NMR}\left(\mathrm{CDCl}_{3}, 50 \mathrm{MHz}\right): \delta 139.9(2 \times \mathrm{C}), 128.7(4 \times \mathrm{CH}), 127.9(4 \times \mathrm{CH}), 126.6(2$ $\times \mathrm{CH}), 71.4(\mathrm{CH}), 56.8(\mathrm{CH}), 54.6\left(2 \times \mathrm{CH}_{2}\right), 44.9\left(\mathrm{CH}_{2}\right), 35.5\left(\mathrm{CH}_{2}\right), 25.2(\mathrm{CH}), 23.2\left(\mathrm{CH}_{3}\right)$, $22.7\left(\mathrm{CH}_{3}\right)$. ESI-MS m/z: $327[\mathrm{M}+\mathrm{H}]^{+}$; MS/MS m/z (rel. int.): 310(100), 233(45), 198(30).

(2R,3S,4S)-1-Amino-3-dibenzylamino-4-methylhexan-2-ol (13e). Colorless oil. 77\% yield. FTIR (neat): 3345, 3289, 3060, 2955, 1601, 1376, 1061, $1027 \mathrm{~cm}^{-1} .{ }^{1} \mathrm{H}$ NMR $\left(\mathrm{CDCl}_{3}, 200\right.$ MHz): $\delta 7.24(\mathrm{~m}, 10 \mathrm{H}), 3.75(\mathrm{~d}, J=13.2 \mathrm{~Hz}, 2 \mathrm{H}), 3.73(\mathrm{~m} 2 \mathrm{H}), 3.63(\mathrm{~s}, 1 \mathrm{H}), 3.54(\mathrm{~d}, J=13.2$ Hz, 2H), 2.65 (dd, $J=13.6,7.0 \mathrm{~Hz}, 1 \mathrm{H}), 2.44$ (dd, $J=7.6,4.6 \mathrm{~Hz}, 1 \mathrm{H}), 2.24$ (m, 1H), 2.03 (br s, $2 \mathrm{H}), 1.90(\mathrm{~m}, 1 \mathrm{H}), 1.65(\mathrm{~m}, 1 \mathrm{H}), 1.08(\mathrm{~d}, J=6.6 \mathrm{~Hz}, 3 \mathrm{H}), 0.95(\mathrm{t}, J=7.6 \mathrm{~Hz}, 3 \mathrm{H}) .{ }^{13} \mathrm{C} \mathrm{NMR}$ $\left(\mathrm{CDCl}_{3}, 50 \mathrm{MHz}\right): \delta 139.7(2 \times \mathrm{C}), 128.8(4 \times \mathrm{CH}), 128.0(4 \times \mathrm{CH}), 126.8(2 \times \mathrm{CH}), 70.1(\mathrm{CH})$, $62.5(\mathrm{CH}), 54.8\left(2 \times \mathrm{CH}_{2}\right), 44.6\left(\mathrm{CH}_{2}\right), 32.4(\mathrm{CH}), 29.8\left(\mathrm{CH}_{2}\right), 16.1\left(\mathrm{CH}_{3}\right), 12.2\left(\mathrm{CH}_{3}\right)$. ESI-MS $m / z: 327[\mathrm{M}+\mathrm{H}]^{+} ; \mathrm{MS} / \mathrm{MS} m / z$ (rel. int.): 309(100), 292(10), 198(35), 91(5). 


\section{General procedure for the synthesis of 1,3-oxazolidin-2-ones 1}

To a solution of amino alcohol 13 in dry $\mathrm{CH}_{2} \mathrm{Cl}_{2}$ was added dropwise a solution of triphosgene (1.0 equivalent) in dry $\mathrm{CH}_{2} \mathrm{Cl}_{2}$ at $0{ }^{\circ} \mathrm{C}$. Then, the reaction mixture was stirred at room temperature for $8 \mathrm{~h}$. A saturated $\mathrm{NaHCO}_{3}$ solution was added and the mixture was stirred for 30 min and then was extracted with dichloromethane $(3 \times 20 \mathrm{~mL})$. The organic layer was separated, dried over $\mathrm{Na}_{2} \mathrm{SO}_{4}$, and filtered. The solvent was evaporated under reduced pressure to give a crude product that was purified by flash chromatography.

(R)-5-((S)-1-Dibenzylamino-2-phenylethyl)-1,3-oxazolidin-2-one (1a). White solid. 73\% yield. Mp $102{ }^{\circ} \mathrm{C}$. $R_{f} 0.50$ (ethyl acetate/petroleum ether $1 / 1$ ); $[\alpha]_{D}^{20}=+8.0^{\circ}$ (c $1.00, \mathrm{CHCl}_{3}$ ). FTIR (KBr): 3283, 3026, 2931, 1754, 1368, $1240 \mathrm{~cm}^{-1} .{ }^{1} \mathrm{H} \mathrm{NMR}\left(\mathrm{CDCl}_{3}, 200 \mathrm{MHz}\right): \delta 7.21(\mathrm{~m}$, 15H), 5.91 (br s, 1H), $4.80(\mathrm{~m}, 1 \mathrm{H}), 3.62$ (br s, 4H), 3.52 (t, $J=8.8 \mathrm{~Hz}, 1 \mathrm{H}), 3.08$ (t, $J=8.8 \mathrm{~Hz}$, 1H), 3.06 (br s, 3H). ${ }^{13} \mathrm{C} \mathrm{NMR}\left(\mathrm{CDCl}_{3}, 50 \mathrm{MHz}\right): \delta 159.6(\mathrm{C}), 139.5(\mathrm{C}), 138.8(2 \times \mathrm{C}), 129.3(2$ $\times \mathrm{CH}), 128.4(4 \times \mathrm{CH}), 128.1(2 \times \mathrm{CH}), 127.9(4 \times \mathrm{CH}), 126.8(2 \times \mathrm{CH}), 126.0(\mathrm{CH}), 76.8(\mathrm{CH})$, $62.4(\mathrm{CH}), 54.4\left(2 \times \mathrm{CH}_{2}\right), 44.8\left(\mathrm{CH}_{2}\right), 32.4\left(\mathrm{CH}_{2}\right)$. ESI-MS m/z: $387[\mathrm{M}+\mathrm{H}]^{+} ; \mathrm{MS} / \mathrm{MS} m / z$ (rel. int.): 326(75), 295(100), 181(40). HPLC: $0.8 \mathrm{~mL} / \mathrm{min} ; 70: 30 \mathrm{MeOH} / \mathrm{H}_{2} \mathrm{O} ; t_{\mathrm{R}}: 6.3 \mathrm{~min}$. HRMS calculated for $\left[\mathrm{C}_{25} \mathrm{H}_{26} \mathrm{~N}_{2} \mathrm{O}_{2}+\mathrm{H}\right]^{+}$387.2073. Found 387.2075.

(R)-5-((S)-1-Dibenzylaminoethyl)oxazolidin-2-one (1b). White solid. $67 \%$ yield. Mp $95^{\circ} \mathrm{C} . R_{f}$ 0.48 (ethyl acetate/petroleum ether 1/1); $[\alpha]_{D}^{20}=+21.2^{\circ}\left(c \quad 1.00, \mathrm{CHCl}_{3}\right)$. FTIR (KBr): 3254, 2826, 1748, $1239 \mathrm{~cm}^{-1} .{ }^{1} \mathrm{H}$ NMR $\left(\mathrm{CDCl}_{3}, 200 \mathrm{MHz}\right): \delta 7.25(\mathrm{~m}, 10 \mathrm{H}), 5.84$ (br s, $\left.1 \mathrm{H}\right), 4.46(\mathrm{q}, J$ $=8.6 \mathrm{~Hz}, 1 \mathrm{H}), 3.70(\mathrm{~d}, J=13.6 \mathrm{~Hz}, 2 \mathrm{H}), 3.54(\mathrm{t}, J=8.6 \mathrm{~Hz}, 1 \mathrm{H}), 3.43(\mathrm{~d}, J=13.6 \mathrm{~Hz}, 2 \mathrm{H})$, $3.23(\mathrm{t}, J=8.6 \mathrm{~Hz}, 1 \mathrm{H}), 2.82(\mathrm{dd}, J=8.6,6.6 \mathrm{~Hz}, 1 \mathrm{H}), 1.22(\mathrm{~d}, J=6.6 \mathrm{~Hz}, 3 \mathrm{H}) .{ }^{13} \mathrm{C} \mathrm{NMR}$ $\left(\mathrm{CDCl}_{3}, 50 \mathrm{MHz}\right): \delta 159.7(\mathrm{C}), 138.8(2 \times \mathrm{C}), 128.7(4 \times \mathrm{CH}), 128.1(4 \times \mathrm{CH}), 127.0(2 \times \mathrm{CH})$, $78.3(\mathrm{CH}), 56.5(\mathrm{CH}), 54.4\left(2 \times \mathrm{CH}_{2}\right), 44.7\left(\mathrm{CH}_{2}\right), 8.6\left(\mathrm{CH}_{3}\right)$. ESI-MS m/z: $311[\mathrm{M}+\mathrm{H}]^{+} ; \mathrm{MS} / \mathrm{MS}$ $m / z$ (rel. int.): 250(50), 219(100), 181(93), 91(60). HPLC: $0.8 \mathrm{~mL} / \mathrm{min} ; 70: 30 \mathrm{MeOH} / \mathrm{H}_{2} \mathrm{O} ; t_{\mathrm{R}}$ : $5.8 \mathrm{~min}$. HRMS calculated for $\left[\mathrm{C}_{19} \mathrm{H}_{22} \mathrm{~N}_{2} \mathrm{O}_{2}+\mathrm{H}\right]^{+} 311.1760$. Found 311.1758 .

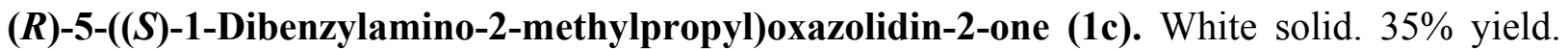
Mp $45^{\circ} \mathrm{C} . R_{f} 0.55$ (ethyl acetate/petroleum ether $1 / 1$ ); $[\alpha]_{D}^{20}=-9.0^{\circ}\left(c 1.00, \mathrm{CHCl}_{3}\right)$. FTIR (KBr): 3284, 2958, 1752, 1239, $1078 \mathrm{~cm}^{-1} .{ }^{1} \mathrm{H} \mathrm{NMR}\left(\mathrm{CDCl}_{3}, 200 \mathrm{MHz}\right): \delta 7.25(\mathrm{~m}, 10 \mathrm{H}), 5.73($ br s, $1 \mathrm{H}), 4.83$ (q, $J=8.6 \mathrm{~Hz}, 1 \mathrm{H}), 3.67$ (d, $J=13.4 \mathrm{~Hz}, 2 \mathrm{H}), 3.62$ (t, $J=8.6 \mathrm{~Hz}, 1 \mathrm{H}), 3.55(\mathrm{~d}, J=$ $13.4 \mathrm{~Hz}, 2 \mathrm{H}), 3.23(\mathrm{t}, J=8.6 \mathrm{~Hz}, 1 \mathrm{H}), 2.58(\mathrm{dd}, J=8.6,3.2 \mathrm{~Hz}, 1 \mathrm{H}), 2.33(\mathrm{~m}, 1 \mathrm{H}), 1.13(\mathrm{~d}, J=$ $7.4 \mathrm{~Hz}, 3 \mathrm{H}), 1.09(\mathrm{~d}, J=7.4 \mathrm{~Hz}, 3 \mathrm{H}) .{ }^{13} \mathrm{C} \mathrm{NMR}\left(\mathrm{CDCl}_{3}, 50 \mathrm{MHz}\right): \delta 159.7(\mathrm{C}), 138.8(2 \times \mathrm{C})$, $128.7(4 \times \mathrm{CH}), 128.1(4 \times \mathrm{CH}), 127.0(2 \times \mathrm{CH}), 75.4(\mathrm{CH}), 64.1(\mathrm{CH}), 54.6\left(2 \times \mathrm{CH}_{2}\right), 45.5$ $\left(\mathrm{CH}_{2}\right), 25.1\left(\mathrm{CH}_{2}\right), 23.3\left(\mathrm{CH}_{3}\right), 19.0\left(\mathrm{CH}_{3}\right)$. ESI-MS m/z: $339[\mathrm{M}+\mathrm{H}]^{+} ; \mathrm{MS} / \mathrm{MS} \mathrm{m} / z$ (rel. int.): 283(100), 247(40), 188(45). HPLC: $0.8 \mathrm{~mL} / \mathrm{min} ; 70: 30 \mathrm{MeOH} / \mathrm{H}_{2} \mathrm{O} ; t_{\mathrm{R}}$ : $5.8 \mathrm{~min}$. HRMS calculated for $\left[\mathrm{C}_{21} \mathrm{H}_{26} \mathrm{~N}_{2} \mathrm{O}_{2}+\mathrm{H}\right]^{+}$339.2073. Found 339.2071.

(R)-5-((S)-1-Dibenzylamino-3-methylbutyl)-1,3-oxazolidin-2-one (1d). White solid. 62\% yield. Mp $43{ }^{\circ} \mathrm{C} . R_{f} 0.52$ (ethyl acetate/petroleum ether $1 / 1$ ); $[\alpha]_{D}^{20}=-30.9^{\circ}\left(c 1.00, \mathrm{CHCl}_{3}\right)$. FTIR (KBr): 3277, 3028, 1753, 1240, $1080 \mathrm{~cm}^{-1} .{ }^{1} \mathrm{H} \mathrm{NMR}\left(\mathrm{CDCl}_{3}, 200 \mathrm{MHz}\right): \delta 7.27$ (m, 10H), 6.09 
(br s, 1H), $4.75(\mathrm{~m}, 1 \mathrm{H}), 3.72(\mathrm{~d}, J=13.8 \mathrm{~Hz}, 2 \mathrm{H}), 3.58(\mathrm{t}, J=8.4 \mathrm{~Hz}, 1 \mathrm{H}), 3.55(\mathrm{~d}, J=13.8$ $\mathrm{Hz}, 2 \mathrm{H}), 3.12$ (t, $J=8.4 \mathrm{~Hz}, 1 \mathrm{H}), 2.67$ (q, $J=7.0 \mathrm{~Hz}, 1 \mathrm{H}), 1.96$ (hpt, $J=6.6 \mathrm{~Hz}, 1 \mathrm{H}), 1.73$ (q, $J$ $=7.0 \mathrm{~Hz}, 1 \mathrm{H}), 1.27(\mathrm{~m}, 1 \mathrm{H}), 0.92(\mathrm{~d}, J=6.6 \mathrm{~Hz}, 3 \mathrm{H}), 0.73(\mathrm{~d}, J=6.2 \mathrm{~Hz}, 3 \mathrm{H}) .{ }^{13} \mathrm{C} \mathrm{NMR}$ $\left(\mathrm{CDCl}_{3}, 50 \mathrm{MHz}\right): \delta 159.9(\mathrm{C}), 139.2(2 \times \mathrm{C}), 128.6(4 \times \mathrm{CH}), 128.0(4 \times \mathrm{CH}), 126.9(2 \times \mathrm{CH})$, $76.8(\mathrm{CH}), 58.2(\mathrm{CH}), 54.4\left(2 \times \mathrm{CH}_{2}\right), 44.9\left(\mathrm{CH}_{2}\right), 35.1\left(\mathrm{CH}_{2}\right), 24.7(\mathrm{CH}), 23.3\left(\mathrm{CH}_{3}\right), 22.4$ $\left(\mathrm{CH}_{3}\right)$. ESI-MS m/z: 353 [M+H] ${ }^{+}$; MS/MS m/z (rel. int.): 261(100), 202(75), 181(80). HPLC: 0.8 $\mathrm{mL} / \mathrm{min} ; 70: 30 \mathrm{MeOH} / \mathrm{H}_{2} \mathrm{O} ; t_{\mathrm{R}}: 6.8 \mathrm{~min}$. HRMS calculated for $\left[\mathrm{C}_{22} \mathrm{H}_{28} \mathrm{~N}_{2} \mathrm{O}_{2}+\mathrm{H}\right]^{+} 353.2229$. Found 353.2226.

(R)-5-((1S,2S)-1-Dibenzylamino-2-methylbutyl)oxazolidin-2-one (1e). White solid. 66\% yield. Mp $42{ }^{\circ} \mathrm{C} . R_{f} 0.62$ (ethyl acetate/petroleum ether $1 / 1$ ); $[\alpha]_{D}^{20}=+11.5^{\circ}$ (c $1.00, \mathrm{CHCl}_{3}$ ). FTIR (KBr): 3277, 2961, 1752, 1237, $1077 \mathrm{~cm}^{-1} .{ }^{1} \mathrm{H}$ NMR $\left(\mathrm{CDCl}_{3}, 200 \mathrm{MHz}\right): \delta 7.27(\mathrm{~m}, 10 \mathrm{H})$, 5.49 (br s, $1 \mathrm{H}), 4.86(\mathrm{q}, J=8.4 \mathrm{~Hz}, 1 \mathrm{H}), 3.73(\mathrm{~d}, J=13.6 \mathrm{~Hz}, 2 \mathrm{H}), 3.66(\mathrm{t}, J=8.4 \mathrm{~Hz}, 1 \mathrm{H}), 3.48$ $(\mathrm{d}, J=13.6 \mathrm{~Hz}, 2 \mathrm{H}), 3.26(\mathrm{t}, J=8.4 \mathrm{~Hz}, 1 \mathrm{H}), 2.66(\mathrm{dd}, J=9.0,1.6 \mathrm{~Hz}, 1 \mathrm{H}), 2.03(\mathrm{~m}, 1 \mathrm{H}), 1.44$

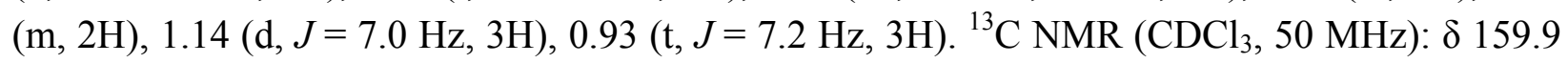
(C), $139.2(2 \times \mathrm{C}), 129.0(4 \times \mathrm{CH}), 128.4(4 \times \mathrm{CH}), 127.3(2 \times \mathrm{CH}), 75.4(\mathrm{CH}), 63.6(\mathrm{CH}), 54.7$ $\left(2 \times \mathrm{CH}_{2}\right), 45.7\left(\mathrm{CH}_{2}\right), 31.7(\mathrm{CH}), 30.0(\mathrm{CH}), 15.6\left(\mathrm{CH}_{3}\right), 12.4\left(\mathrm{CH}_{3}\right)$. ESI-MS m/z: $353[\mathrm{M}+\mathrm{H}]^{+}$; MS/MS m/z (rel. int.): 283(100), 202(45). HPLC: $0.8 \mathrm{~mL} / \mathrm{min} ; 70: 30 \mathrm{MeOH} / \mathrm{H}_{2} \mathrm{O} ; t_{\mathrm{R}}: 8.4 \mathrm{~min}$. HRMS calculated for $\left[\mathrm{C}_{22} \mathrm{H}_{28} \mathrm{~N}_{2} \mathrm{O}_{2}+\mathrm{H}\right]^{+}$353.2229. Found 353.2225.

\section{Acknowledgements}

We gratefully acknowledge support for this project from Consejo Nacional de Ciencia y Tecnología (CONACyT, GRANT No. SEP-2004-CO1-47835).

\section{References}

1. Evans, D. A.; Bartroli, J.; Shih, T. L. J. Am. Chem. Soc. 1981, 103, 2127.

2. For a review of N-acyloxazolidinones applications see: (a) Evans, D. A. Aldrichimica Acta 1982, 15, 23. (b) Ager, D. J.; Prakash, I.; Schaad, D. R. Chem. Rev. 1996, 96, 835. (c) Ager, D. J.; Prakash, I.; Schaad, D. R. Aldrichimica Acta 1997, 30, 3. (d) Hintermann, T.; Seebach, D. Helv. Chim. Acta 1998, 81, 2093. (e) Fukuzawa, S.; Matsuzawa, H.; Yoshimitsu, S. J. Org. Chem. 2000, 65, 1702.

3. (a) Brickner, S. J.; Barbachyn, M. R.; Hutchinson, D. K.; Manninen, P. R. J. Med. Chem. 2008, 51, 1981. (b) Perry, C. M.; Javis, B. Drugs 2001, 61, 525.

4. (a) Means, J. A.; Katz, S.; Nayek, A.; Anupm, R.; Hines, J. V.; Bergmeier, S. C. Bioorg. Med. Chem. Lett. 2006, 16, 3600. (b) Bozdogan, B.; Appelbaum, P. C. Int. J. Antimicrob. Agents 2004, 23, 113. (c) Kloss, P.; Xiong, L.; Shinabarger, D. L.; Mankin, A. S. J. Mol. Biol. 1999, 294, 103. 
5. (a) Clemmet, D.; Markham, A. Drugs 2000, 59, 815. (b) Shinaberger, D. L.; Marotti, K. R.; Murray, R. W.; Lin, A. H.; Melchoir, E. P.; Swaney, S. M.; Dunyak, D. S.; Demyan, W. F.; Buysse, J. M. Antimicrob. Agents Chemother. 1997, 41, 2132.

6. (a) Ebner, D. C.; Culhane, J. C.; Winkelman, T. N.; Haustein, M. D.; Ditty, J. L.; Ippoliti, J. T. Bioorg. Med. Chem. 2008, 16, 2651. (b) Takhi, M.; Murugan, C.; Munikumar, M.; Bhaskarreddy, K. M.; Singh, G.; Sreenivas, K.; Sitaramkumar, N.; Das, J.; Trehan, S.; Iqbal, J. Bioorg, Med. Chem. Lett. 2006, 16, 2391. (c) Renslo, A. R.; Luehr, G. W.; Gordeev, M. F. Bioorg. Med. Chem. 2006, 14, 4227. (d) Pallavicini, M.; Moroni, B.; Bolchi, C.; Cilia, A.; Clementi, F.; Fumagalli, L.; Gotti, C.; Meneghetti, F.; Raganti, L.; Vistoli, G.; Valoti, E. Bioorg. Med. Chem. Lett. 2006, 16, 5610. (e) Das, J.; Sitaram-Kumar, M.; Subrahmanyam, D.; Sastry, T. V. R. S.; Prasad-Narasimhulu, C.; Laxman-Rao, C. V.; Kannan, M.; Roshaiah, M.; Awasthi, R.; Patil, S. N.; Sarnaik, H. M.; Rao-Mamidi, N. V. S.; Selvakumar, N.; Iqbal, J. Bioorg. Med. Chem. 2006, 14, 8032. (f) Wang, G.; Ella-Menye, J. R.; Sharma, V. Bioorg. Med. Chem. Lett. 2006, 16, 2177. (g) Phillips, O. A.; Udo, E. E.; Ali, A. A. M.; Al-Hassawi, N. Bioorg. Med. Chem. 2003, 11, 35.

7. Negwer, M.; Scharnow, H. G. Organic Chemical Drugs and Their Synonyms, $8^{\text {th }}$ Ed.; WileyVCH: Weinheim, Germany, 2001, p 2760.

8. (a) Mapp, Y.; Nodine, J. H. Pyschosomatics 1962, 3, 458. (b) Kraft, I. A. Am. J. Psychiatry 1962, 118, 841. (c) Lowinger, P. Am. J. Psychiatry 1963, 120, 66. (d) Eskenazi, J.; Nikiforidis, T.; Livio, J. J.; Schelling, J. L. Europ. J. Clin. Pharmacol. 1976, 9, 411. (e) Wuis, E. W. Pharm. Weekbl. Sci. Ed. 1987, 9, 249.

9. (a) Mai, A.; Artico, M.; Esposito, M.; Ragno, R.; Sbardella, G.; Massa, S. Il Farmaco 2003, 58, 231. (b) Wouters, J.; Moureau, F.; Evrard, G.; Koening, J. J.; Jegham, S.; George, P.; Durant, F. Bioorg. Med. Chem. 1999, 7, 1683. (c) Dostert, P.; Strolin-Benedetti, M.; Tipton, K. F. Med. Res. Rev. 1989, 9, 45. (d) Kan, J. P.; Pujol, J. F.; Malnoe, A.; Strolin-Benedetti, M.; Gouret, C.; Raynaud, G. Eur. J. Med. Chem. Chim. Ther. 1977, 12, 13.

10. (a) Morán-Ramallal, R.; Liz, R.; Gotor, V. Org. Lett. 2008, 10, 1935. (b) Madhusudhan, G.; Om-Reddy, G.; Rajesh, T.; Ramanathan, J.; Dubey, P. K. Tetrahedron Lett. 2008, 49, 3060. (c) Ella-Menye, J. R.; Wang, G. Tetrahedron 2007, 63, 10034. (d) Li, P.; Yuan, X.; Wang, S.; Lu, S. Tetrahedron 2007, 63, 12419. (e) Nguyen-Le, T.; Bao-Nguyen, Q. P.; NyoungKim, J.; Hyeom-Kim, T. Tetrahedron Lett. 2007, 48, 7834. (f) Zappia, G.; Gacs-Baitz, E.; Delle-Monache, G.; Misiti, D.; Nevola, L.; Botta, B. Curr. Org. Synth. 2007, 4, 81. (g) Wang, G.; Ella-Menye, J. R.; Sharma, V. Bioorg. Med. Chem. Lett. 2006, 16, 2177. (h) Hein, J. E.; Geary, L. M.; Jaworsky, A. A.; Hultin, P. G. J. Org. Chem. 2005, 70, 9940.

11. (a) Reetz, M. T. Chem. Rev. 1999, 99, 1121. (b) Reetz, M. T. Pure Appl. Chem. 1992, 64, 351. (c) Reetz, M. T. Angew. Chem. Int. Ed. Engl. 1991, 30, 1531. (d) Reetz, M. T.; Drewes, M. W.; Harms, K.; Reif, W. Tetrahedron Lett. 1988, 29, 3295.

12. (a) Andrés, J. M.; Martínez, M. A.; Pedrosa, R.; Pérez-Encabo, A. Tetrahedron: Asymmetry 2001, 12, 347. (b) Manickam, G.; Nogami, H.; Kanai, M.; Gröger, H.; Shibaski, M. Synlett 2001, 5,617 . 
13. (a) Nogami, H.; Kanai, M.; Shibasaki, M. Chem. Pharm. Bull. 2003, 51, 702. (b) Bernardi, L.; Bonini, B. F.; Dessole,G.; Fochi, M.; Comes-Franchini, M.; Gavioli, S.; Ricci, A.; Varchi, G. J. Org. Chem. 2003, 68, 1418.

14. (a) Li, P.; Yuan, X.; Wang, S.; Lu, S. Tetrahedron 2007, 63, 12419. (b) Tiecco, M.; Testaferri, L.; Temperini, A.; Bagnoli, L.; Marini, F.; Santi, C. Chem. Eur. J. 2004, 10, 1752.

15. Ochoa-Terán, A.; Rivero, I. A. ARKIVOC 2008, (ii), 235.

16. Concellón, J. M.; Riego, E.; Rivero, I. A.; Ochoa, A. J. Org. Chem. 2004, 69, 6244.

17. (a) Reetz, M. T.; Drewes, M. W.; Schmitz, A. Angew. Chem. Int. Ed. 1987, 26, 1141. (b) Taoues, L.; Chastanet, J.; Zhu, J. J. Org. Chem. 1998, 63, 1709. 